\title{
AvaGames: Um Ambiente para Apoio a Avaliação da Aprendizagem em Jogos Digitais
}

\author{
Enza Rafaela De Nadai Victal ${ }^{1}$, Crediné Silva de Menezes ${ }^{2}$ \\ ${ }^{1}$ Departamento de Informática - Universidade Federal do Espírito Santo (UFES) Caixa \\ Postal 101.9011 - 29.075-910 - Vitória - ES - Brasil
}

${ }^{2}$ Faculdade de Educação - Universidade Federal do Rio Grande do Sul Av. Paulo Gama, s/n, Farroupilha - Porto Alegre - RS - Brasil - Caixa Postal 90046-900

\{rafaeladenadai,credine\}@gmail.com

\begin{abstract}
Aware of the digital games relevance as a tool to enhance education, we bring up of the need to have a wide perception of what happens in a game. The learning evaluation, of fundamental importance in the educational processes, constitutes a great challenge in the context of digital use. There are many evidences of learning possibilities through the use of games, obtained by researches, however, when a teacher wishes to assess what a particular student has learned, still resents the lack of tools that provide support. In this work, we present a computing environment for gathering evidences and data analysis support.
\end{abstract}

Resumo: Cientes da relevância dos jogos digitais como ferramentas para potencializar a educação, levanta-se a questão da necessidade de ter uma percepção mais ampla do que acontece dentro de um jogo. A avaliação da aprendizagem, de fundamental importância nos processos educacionais, constitui-se em um grande desafio no contexto do uso de recursos digitais. Há bastantes evidências das possibilidades de aprendizagem com o uso de jogos, obtidos através de pesquisas, entretanto, quando um professor deseja avaliar o que um determinado aluno aprendeu, ainda se ressente a ausência de instrumentos que lhe ofereçam apoio. Neste trabalho apresentamos um ambiente para coleta de evidencias e suporte à análise dos dados coletados.

\section{Introdução}

Os jogos são uma parte da experiência humana que está presente em todas as culturas. Crawford (1984) vincula a ideia de arte na definição de um jogo, sendo a arte algo projetado para gerar emoção através da fantasia. O autor ainda complementa que a interação é a maior e mais completa forma de representação de um jogo.

Gee (2007) também ressalta que "os bons videogames ${ }^{1}$ incorporam bons princípios de aprendizagem, princípios apoiados pelas pesquisas atuais em Ciência Cognitiva". E devido à capacidade do que proporcionam, jogos digitais passaram a ser vistos com muito potencial de uso na educação, justamente pelo fato de serem

\footnotetext{
${ }^{1}$ Jogo eletrônico que envolve interações entre jogador e imagens enviadas à um dispositivo que as exibe (televisão ou monitor).
} 
ambientes atrativos e interativos, que prendem a atenção de quem joga a partir do momento em que desafios são oferecidos.

Contudo, não é suficiente apenas disponibilizá-los em sala de aula como uma ferramenta de apoio à aprendizagem. É necessário saber o que de fato o aluno está aprendendo a partir deste jogo. E para que isso ocorra, o mais indicado é ter registros de eventos durante os jogos através do próprio computador. $\mathrm{O}$ processamento dos dados permite gerar dados analíticos constantes, possibilitando apoiar a avaliação. Com isso, apresentamos neste artigo um ambiente para apoiar o processo de monitoramento e elaboração da avaliação em jogos digitais.

Este artigo está organizado da seguinte forma: a Seção seguinte contém a metodologia de pesquisa utilizada. Na Seção 3 apresenta-se uma aproximação para avaliação, com intuito de correlacionar a importância e os elementos de percepção do jogo com a avaliação. Na Seção 4 retratamos um pouco sobre a problemática que nos motivou a pesquisa. A Seção 5 expõe os trabalhos correlatos. E por fim, as Seções 6 e 7 apresentam uma proposta de solução para o problema exposto na Seção 4, seguida das considerações finais na Seção 8.

\section{Metodologia de Pesquisa}

Incialmente procedeu-se uma revisão sistemática da literatura, para aprofundamento sobre elementos centrais - como "jogos digitais", "aprendizagem" e "avaliação" - para saber o que outros pesquisadores estavam produzindo neste assunto, identificando assim o problema de pesquisa. Foi realizado um amplo levantamento do estado da arte, através de artigos que mais se aproximaram do contexto desejado, os trabalhos correlatos que são citados na Seção 5, que contribuíram para elaboração de uma nova proposta, apresentada neste artigo.

Quando se trata de avaliação, se ressente a carência de recursos que apoiem a utilização de jogos digitais em sala de aula e após verificar que haveria campo para uma nova proposta, formulamos um sistema para apoiar a avaliação de jogos. Todo processo de concepção desse sistema compreendeu a modelagem através de diagramas, elaboração de arquitetura e aplicação para uma validação preliminar, com alunos e professor. O principal objetivo desse sistema é apoiar o agente avaliador a estruturar a avaliação com base em dados gerados a partir de um jogo digital.

\section{A Avaliação da Aprendizagem em Jogos Digitais}

Em sala de aula, o professor está constantemente à procura de sinais que possam permitir que ele perceba o nível de compreensão dos alunos. Em ambientes virtuais, neste caso, jogos digitais, que vem ocupando cada vez mais o seu devido espaço para sessões de aprendizagens, existe uma necessidade ainda maior.

Entretanto, Rupp et al. (2010) iniciaram suas pesquisas no assunto constatando que não existem normas disciplinares exatas que evidencie validade na avaliação em jogos. No entanto, existem diretrizes para pensar sobre a qualidade das evidências no contexto de um. Tais diretrizes fazem parte de um modelo conhecido como Evidence Centered Design, o ECD. O objetivo é, através de um esboço de jogo, investigar a influência de dados que são recolhidos durante o jogar e como esses dados podem ser analisados para identificar adequadamente o desenvolvimento de competências de diferentes alunos. 
V Congresso Brasileiro de Informática na Educação (CBIE 2016)

Anais dos Workshops do V Congresso Brasileiro de Informática na Educação (CBIE 2016)

\section{Problematização: a Falta de Suporte para Avaliação em Jogos Digitais}

O processo de uma sessão de jogo é longo, rico e complexo e, não pode ser analisado apenas pela chegada ao final do jogo com certa quantidade de pontos acumulados. Ao utilizar um jogo para apoiar as atividades em sala de aula, caso o professor note uma ação específica, ele faz uma anotação e pode fazer essa observação ali mesmo, em tempo real ou aguardar o fim do jogo para dar um feedback.

A observação humana, nesse caso, depara-se com várias dificuldades, das quais destacamos a necessidade de que seja jogado na presença do observador, o observador precisa estar completamente focado para fazer suas anotações e também há a limitação na quantidade de observadores.

E a partir do problema, surgiu o propósito desta pesquisa, que é apresentar uma contribuição que apoie o processo de acompanhamento, percepção e, claro, avaliação a partir de dados gerados em um jogo.

\section{Trabalhos Correlatos}

Apresentamos nessa seção os trabalhos que foram selecionados no levantamento do estado da arte dessa pesquisa.

\section{a) Concepção Multiagente e Automatização da Observação}

Durante suas atividades, os alunos deixam o que Carron et al. (2006) define como "rastros". E o interesse está na observação dos comportamentos dos alunos em ambientes de aprendizagem, com base nesses rastros, a fim de fornecer ao professor informações para que ele possa reagir a situações particulares. Carron et al. (2006) então utilizou um sistema multiagente, estruturando os agentes da seguinte maneira: Agente Coletor (Collector), considerando que cada aluno trabalhe em uma estação, cada máquina a ser observada precisa de um agente localmente para coletar os dados; Agente de Estruturação (Structuring), este agente dedica-se a encontrar padrões em vestígios recolhidos para melhor abstração dos dados coletados; Agente de Visualização (Viz), ajuda a interpretar os vestígios, dedicando-se à sua visualização. Os agentes interagem entre si e durante um jogo o professor pode querer ter uma visão sobre as atividades dos alunos, solicitar e também configurar os agentes para avisá-lo de algum comportamento específico identificado nas estações dos alunos.

\section{b) Integração da Plataforma $<e$-Adventure $>$ e LAMS}

Del Blanco et al. (2010) apresentam em sua pesquisa, a integração dos jogos educacionais criados na plataforma $<e$-Adventure $>^{2}$ com LAMS (Sistemas de Gerenciamento da Atividade de Aprendizagem). Esta integração ajuda ao professor usar a informação recolhida durante um jogo e conduzir o aluno através de diferentes atividades de aprendizagem ou simplesmente recolher mais informações que podem ser utilizadas para outros fins de avaliação e monitoramento. O principal objetivo é para proporcionar uma solução de aprendizagem global que facilita o uso de jogos durante todo o processo de ensino-aprendizagem.

\section{c) EngAGe: um Motor de Avaliação para Jogos}

\footnotetext{
${ }^{2}$ Uma plataforma de criação de jogos e simulações para fins pedagógicos, disponível na Internet e que não requer habilidades de programação tão aprofundadas para conceber os jogos.
} 
V Congresso Brasileiro de Informática na Educação (CBIE 2016)

Anais dos Workshops do V Congresso Brasileiro de Informática na Educação (CBIE 2016)

Chaudy, Connolly and Hainey (2014) apresentam em sua pesquisa um engine (motor) de avaliação chamado EngAGe. Um dos objetivos do EngAGe é fornecer aos desenvolvedores de jogos e professores uma ferramenta de avaliação, bem como orientações sobre as características de avaliação que devem existir no jogo. $\mathrm{O}$ engine também permite separar a mecânica do jogo da lógica de avaliação, tornando assim o sistema mais flexível. As especificações do EngAGe incluem: uma DSL (DomainSpecific Language, ou linguagem de domínio específico) para ser utilizada como um descritor de configuração avaliação; um conjunto de serviços web para analisar a DSL e realizar a avaliação. Através do relatório de análise de aprendizagem, um professor pode descobrir, por exemplo, que a tarefa é demasiadamente difícil ou ambígua, se a maioria dos jogadores entendeu errado.

\subsection{Análise de Trabalhos Correlatos}

Todas as considerações feitas de cada trabalho estão em resumo na Tabela 1, ressaltando as particularidades de cada pesquisa. Essas considerações serviram de contribuição para elaboração da proposta de solução e também na identificação de funcionalidades complementares, ainda inexistentes nos trabalhos já elaborados.

Tabela 1 - Síntese de Trabalhos Correlatos e suas características.

\begin{tabular}{|c|c|c|}
\hline Correlato & Tecnologia & Diferenciais \\
\hline $\begin{array}{l}\text { Concepção } \\
\text { Multiagente e } \\
\text { Automatização da } \\
\text { Observação } \\
\text { (Carron et al., 2006) }\end{array}$ & $\begin{array}{l}\text { Sistemas Multiagente } \\
\text { desenvolvido em } \\
\text { linguagem de } \\
\text { programação Java; }\end{array}$ & $\begin{array}{l}\text { - Os agentes estão em camadas, cada um com uma } \\
\text { função específica e definida; } \\
\text { - O agente observador oferece opção de ser calibrado } \\
\text { pelo avaliador; } \\
\text { - São gerados LOGs legíveis para o avaliador, } \\
\text { relacionando o que o professor configurou versus o } \\
\text { que o aluno está fazendo; }\end{array}$ \\
\hline $\begin{array}{l}\text { Integração da } \\
\text { Plataforma }<e \text { - } \\
\text { Adventure }>\text { e LAMS } \\
\text { (Del Blanco et al., } \\
\text { 2010) }\end{array}$ & $\begin{array}{l}\text { Jogo a partir do }<e- \\
\text { Adventure }>\text {; } \\
\text { LAMS. }\end{array}$ & $\begin{array}{l}\text { - Há um jogo que pode ser configurado pelo } \\
\text { professor; } \\
\text { - É definido o chamado "perfil de avaliação", que é } \\
\text { configurado pelo professor; } \\
\text { - Durante o jogo e com base no perfil de avaliação, } \\
\text { são desencadeadas ações, através de atribuições de } \\
\text { variáveis. }\end{array}$ \\
\hline $\begin{array}{l}\text { Engage: um Motor de } \\
\text { Avaliação para Jogos } \\
\text { (Chaudy, Connolly } \\
\text { and Hainey, 2014) }\end{array}$ & $\begin{array}{l}\text { Utilização de DSL; } \\
\text { Serviços web. }\end{array}$ & $\begin{array}{l}\text { - Fornece um arquivo de configuração em DSL; } \\
\text { - O engine preenche a base de dados interna e cria um } \\
\text { conjunto de serviço web especifico para cada jogo, } \\
\text { como uma instância; } \\
\text { - Existe um protocolo de comunicação específico } \\
\text { para executar a avaliação do jogo. }\end{array}$ \\
\hline
\end{tabular}

A partir dessa listagem, pode-se abstrair contribuições dos trabalhos correlatos e apresentar diferenciais, visando melhorias, evoluções e inovações nos ambientes já propostos. Alguns diferenciais são:

- Oferecer a possibilidade de integração com qualquer jogo;

- Oferecer a possibilidade de recuperar dados passados para monitoramento a qualquer hora;

- Utilização desses dados para geração de relatório que apoie a avaliação;

- Disponibilidade de um repositório de jogos e dados de jogo de diversos jogadores; 
V Congresso Brasileiro de Informática na Educação (CBIE 2016)

Anais dos Workshops do V Congresso Brasileiro de Informática na Educação (CBIE 2016)

- Deixar abertura para criação de um jogo inteligente, que se adapte às necessidades de professores e alunos;

\section{AvaGames: um Ambiente de Apoio a Avaliação da Aprendizagem}

Através da problematização da Seção 4, apresentamos o AvaGames, um ambiente de monitoramento e apoio à avaliação, como uma contribuição na solução do problema.

\subsection{Arquitetura AvaGames}

O modelo geral foi dividido em: Ambiente de Jogo e o AvaGames. Dentro do AvaGames há: serviço web para coleta de dados (Serviço de Coleta, Ambiente de Monitoramento e Ambiente de Avaliação). Na Figura 1 abaixo, é apresentada a arquitetura do ambiente proposto. O Ambiente de Jogo, interage com o AvaGames, enviando dados. Para receber esses dados, há um serviço web (web service) com rotinas específicas para esse processo de coleta, armazenando esses dados em uma base. Sendo assim, tanto o Ambiente de Monitoramento como Ambiente de Avaliação podem acessar essa base de dados e recuperar estes dados, seja para visualização ou para iniciar o processo de avaliação.

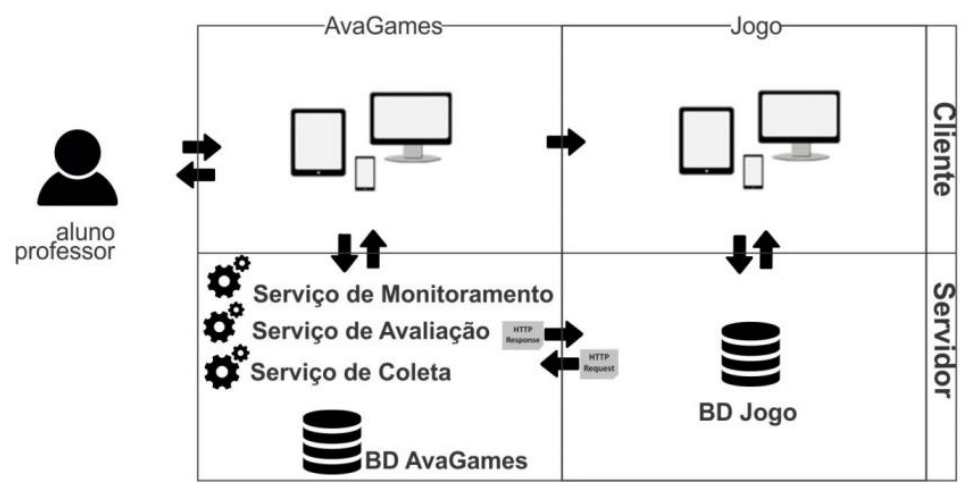

Figura 1 - Arquitetura AvaGames. Fonte: Autoria Própria.

Os principais componentes são descritos a seguir:

- Jogo: pode ser um ambiente externo, ou seja, independente do AvaGames. O jogo envia sequências de requisições para o AvaGames, contendo todos os eventos mapeados;

- Serviço de Coleta: trabalha em background recebendo os dados do jogo. Para isso utilizaremos um web service. Essa parte trabalha com duas camadas: de controle (responsável por ler e tratar os dados recebidos) e a camada de armazenamento (responsável por guardar na base de dados local, essas informações tratadas pela camada de controle);

- Ambiente de Monitoramento: lida especificamente com a camada de interface (que é a apresentação das informações para o usuário), recuperando os dados anteriormente armazenados, apresentando-os de diferentes formas para o usuário, com a possibilidade de acesso por seleção;

- Ambiente de Avaliação: geração de dados analíticos envolvendo os objetivos de aprendizagem, que é customizada pela camada de interface de Avaliação, recuperando os dados anteriormente armazenados; 
Tanto professor, quanto aluno acessam a interface oferecida pelo AvaGames. Nele estarão todos os jogos disponíveis para o professor escolher, ou seja, o jogo sempre estará vinculado ao AvaGames. Portanto, o professor pode escolher e aplicar o jogo que desejar, desde que este esteja disponível.

Assim como a Figura 2 apresenta, a classe Jogo é onde estão cadastrados todos os jogos vinculados ao AvaGames. Quando o professor cria uma Sala, ele vincula o Jogo que será jogado e alunos que irão participar. Formando assim um tipo de repositório de Jogos, que são disponibilizados (se de comum acordo com desenvolvedores do Jogo e AvaGames) para monitoramento e avaliação.

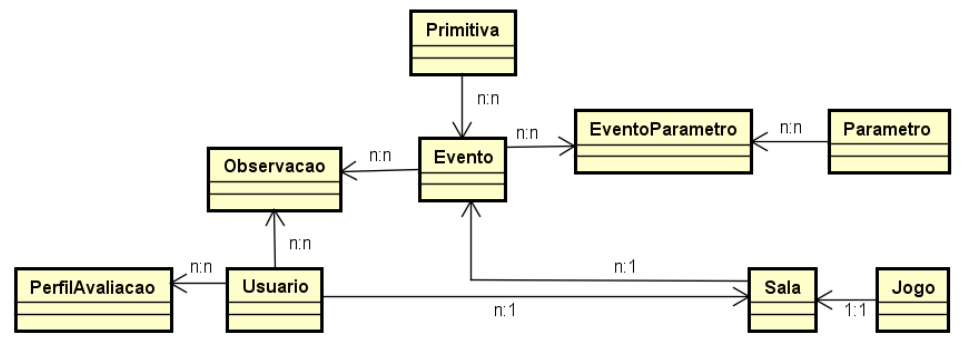

Figura 2 - Diagrama de Classes do AvaGames. Fonte: Autoria Própria.

\section{Registro das Sessões de Jogo}

Esta seção apresenta uma explicação mais detalhada sobre cada parte do AvaGames e seus procedimentos, como é feita a recepção e reconhecimento dos dados vindos do jogo, seu devido armazenamento e como é feita a recuperação desses dados, exemplificando com um jogo.

\subsection{As Primitivas de Monitoramento}

Ressaltando que esse ambiente foi elaborado para receber qualquer evento do jogo, observou-se a necessidade de elaborar uma padronização desses dados a serem registrados. Para isso, foram então criadas as chamadas primitivas de monitoramento.

Uma diversidade grande de eventos pode acontecer durante uma sessão de jogo, entretanto, foram predefinidas algumas primitivas centrais. Isto é, eventos que são possíveis pressupor que serão contemplados nos jogos. Essas primitivas de monitoramento são proveitosas também para envio de dados do jogo para o ambiente de monitoramento, pois, desta forma, facilita o processo de classificação de cada interação do jogo. Por exemplo, quando um jogador inicia o jogo, é acionada a primitiva "inicioujogo" com parâmetros "quem", "onde", "quando" indicando o início de uma sessão de jogo. O mesmo ocorre para indicar o fim de uma sessão, com a primitiva "finalizoujogo", e há também primitivas como "enviou-mensgem", "atribuicao-recursos", "resolveu-problema", entre outros.

\subsection{O Jogo}

Para compreender melhor a relação das primitivas, exemplificaremos com um jogo. O Supermarket Game é um jogo que se passa em um supermercado, onde diversas aventuras são desencadeadas. No jogo pode haver comunicação entre os jogadores e usuário irá experimentar como um passeio em um cenário de supermercado pode ser muito rico em aprendizados. O jogo é linear, onde é dada uma lista de produtos (um 
V Congresso Brasileiro de Informática na Educação (CBIE 2016)

Anais dos Workshops do V Congresso Brasileiro de Informática na Educação (CBIE 2016)

pouco diferente) para o jogador, em forma de missão. Os produtos da lista são gerados pelo sistema, um a um. Assim, para cada missão é possível dividir no jogo o que será trabalhado: Formas, Tamanhos e Cores; Contar, Medir e Classificar; Operações Básicas (somar, diminuir, dividir e subtrair) e contagem de calorias; e assim por diante. $\mathrm{O}$ jogador pode se locomover pela tela principal do jogo, adentrar pelos corredores, correspondentes aos setores do supermercado e ainda pode visualizar outros jogadores e enviar mensagens. A Figura 3 apresenta uma representação da tela principal do SG (Supermarket Game).

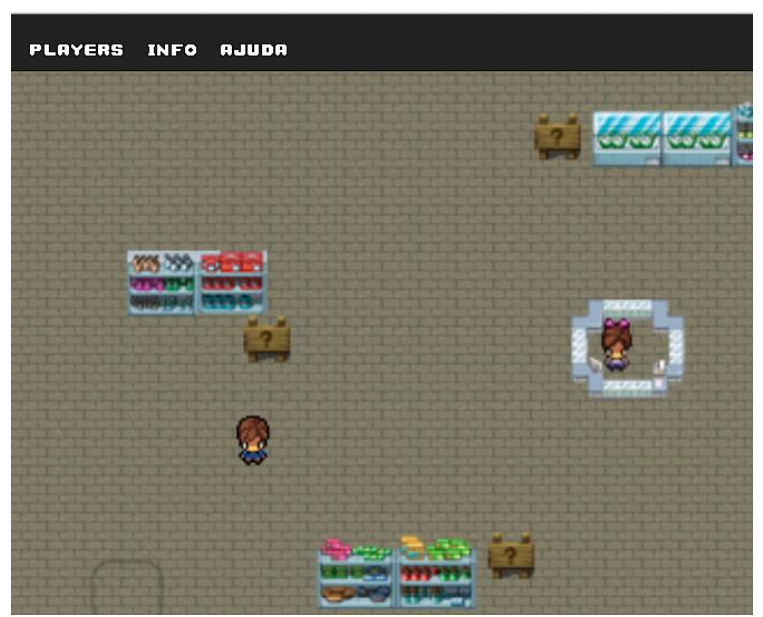

Figura 3 - Tela Principal do Supermarket Game. Fonte: Autoria Própria.

\subsection{Registro}

Quando uma ocorrência é identificada, o jogo envia requisições para o AvaGames. O Serviço de Coleta, que está descrito na arquitetura na Figura 1, recebe um ou mais dados, chamados de requests (request1, request 2 , request 3 e assim sucessivamente). Cada request contém dados que serão lidos pelo Serviço de Coleta.

Essas requests são enviadas utilizando Rest, um protocolo de comunicação baseado no protocolo HTTP cuja maior vantagem é sua flexibilidade, onde o desenvolvedor pode se adaptar ao formato mais adequado de mensagens, de acordo com sua necessidade. Os formatos mais comuns são JSON, XML ou texto puro.

Na Figura 4 um exemplo de request é representa com seu respectivo código, um texto puro utilizando tags para melhor estruturar suas informações. Após processar essas requisições, esses dados são enviados para armazenamento na base de dados. É importante ressaltar dados que são disponibilizado de dentro do AvaGames, são informados na tag "info" na request exemplificada, sendo possível assim identificá-lo no momento da gravação dos registros.

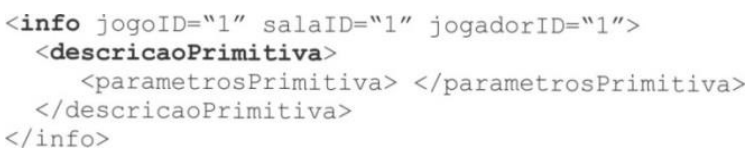

Figura 4 - Exemplo de mensagem recebido no Web Service. Fonte: Autoria Própria. 
V Congresso Brasileiro de Informática na Educação (CBIE 2016)

Anais dos Workshops do V Congresso Brasileiro de Informática na Educação (CBIE 2016)

\subsection{Monitoramento}

Após todo processo de recebimento e gravação de dados do jogo, o avaliador pode decidir ver os dados do jogo. Essa visualização acontece sendo apresentada uma linha do tempo para cada aluno (Figura 5), seguindo uma ordem cronológica. O professor pode ainda realizar observações sobre determinado evento registrado. E assim, o professor consegue consultar estes dados a todo o momento e formular uma avaliação e elucubrar o feedback de uma forma mais abrangente e facilitada, uma vez que não precisaria estar acompanhando pessoalmente um aluno por vez.

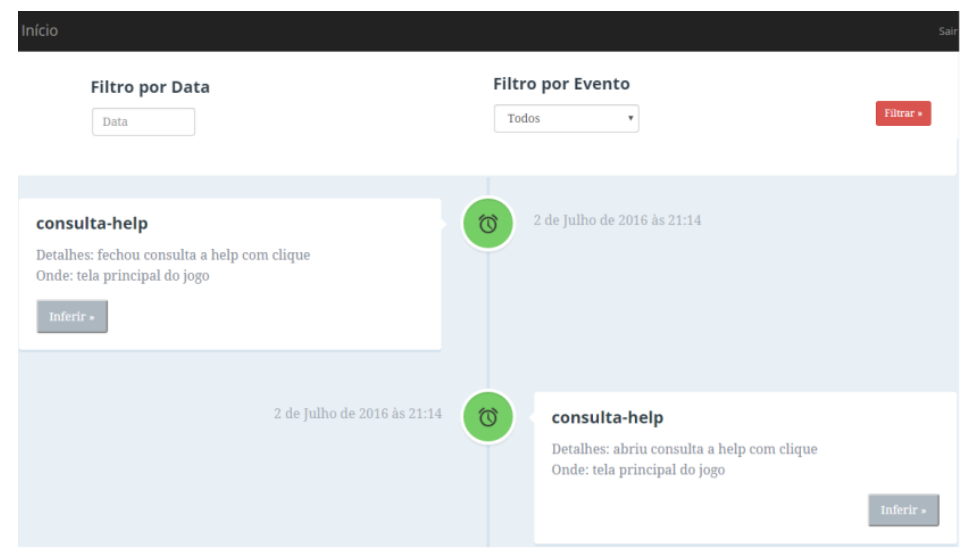

Figura 5 - Timeline Individual. Fonte: Autoria Própria.

\subsection{Avaliação}

O modelo ECD, citado na Seção 3, nos ajudou na estruturação do relatório de avaliação. Incialmente o avaliador precisa identificar variáveis de competência chave de sua avaliação, determinando metas e propósitos da avaliação. Em seguida precisa definir as variáveis no jogo, ou seja, todas as evidências que foram capturadas das sessões do jogo. O avaliador irá relacionar esses dois grupos de variáveis, dizendo qual alcance dos níveis de competências que podem ser medidos no jogo. Toda essa configuração é feita através de uma interface gráfica, gerando assim um perfil de avaliação, que será processado e, como resultado, apresentará os dados para o professor (Figura 6), de forma a facilitar o entendimento do professor sobre esses dados que ele configurou.

\section{Aluno $4 \mathbf{1}$}

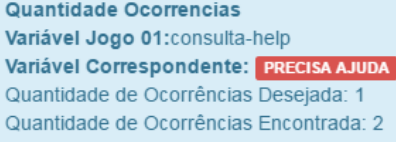

Figura 6 - Resultado do Processamento do Perfil de Avaliação. Fonte: Autoria Própria.

Foi feito ainda uma aplicação preliminar realizada com oito alunos (quatro meninas e quatro meninos) com faixa etária de 10 e 11 anos de idade, equivalente ao quinto ano da educação básica. Com base nas informações do professor, entre as características da turma destacam-se que são participativos, bem informados, possuem senso crítico e com desempenho elevado. Quanto às características sócio econômicas, é uma turma carente, 
V Congresso Brasileiro de Informática na Educação (CBIE 2016)

Anais dos Workshops do V Congresso Brasileiro de Informática na Educação (CBIE 2016)

entretanto, com acesso a recursos tecnológicos e internet. Portanto já havia familiaridade com tecnologia e jogos.

O jogo foi aplicado por cerca de uma hora, onde foi possível capturar uma quantidade razoável de registros gerados pelos alunos, podendo observar também a habilidade de manipular objetos no jogo foi se tornando mais fácil na medida em que os alunos foram se familiarizando com o ambiente.

A aplicação preliminar do um jogo digital em sala de aula serviu pra deixar todo o processo mais proveitoso. A visão que o professor pôde ter sobre o AvaGames, depois de apresentado, explicado e exemplificado foi que: "o AvaGames, se configura como um instrumento de apoio à avaliação para situar o professor num estágio de análise da aprendizagem do aluno no que se refere ao avanço ou na intervenção de seus trabalhos escolares frente no desenvolvimento do currículo escolar".

Analisando as palavras do professor é possível enxergar a aplicação com resultados positivos, pois o experimento causou uma expectativa muito grande por parte dos alunos ao saberem que iriam utilizar recursos tecnológicos e mais ainda com jogos digitais.

\section{Considerações Finais e Direcionamentos para Trabalhos Futuros}

Por meio do ambiente apresentado, espera-se que este possibilite e apoie o professor a ter uma visão mais ampla e detalhada do que o aluno está fazendo dentro do jogo. Isso permite, além do acompanhamento, um ponto essencial no processo avaliativo: o fornecimento de feedback e a realização de inferências no momento ideal durante ou após o jogo. Tanto o aluno quanto professor também podem ver todo um trajeto de jogo após sua realização e, quando desejar, fazer anotações e observações.

Como trabalho futuro, refletimos no privilégio de ter uma grande base de dados populada com informações de ações decorrentes de seções de jogos de uma diversidade de alunos. Poderíamos, por exemplo, aproveitar esses dados para criar um jogo inteligente, onde o jogo "aprende" coisas sobre o jogador com base em identificação de padrões, tornando o jogo adaptativo ou até mesmo criar um jogo configurável, onde o professor e aluno realizam observações e anotações que possam ser utilizadas para reformular e instanciar novos jogos.

\section{Referências}

Carron, T.; Marty, J. C., Heraud, J. M. and France, L. (2006) "Helping the teacher to reorganize tasks in a collaborative learning activity: an agent-based approach", In: 6th International Conference on Advanced Learning Technologies.

Chaudy, Y., Conolly, T. e Hainey, T. (2014) "An Assessment Engine: Educators as Editors of their Serious Games Assessment", In: Busch, European Conference on Games Based Learning. Berlim. Academic Conferences and Publishing International Limited,Reading, UK.

Crawford, C. (1984) "The art of computer game design". Disponível em: <http://www.rohan.sdsu.edu/ stewart/cs583/ACGD_ArtComputerGameDe sign_ChrisCrawford_1982>. Acessado em: Maio 2016. 
V Congresso Brasileiro de Informática na Educação (CBIE 2016)

Anais dos Workshops do V Congresso Brasileiro de Informática na Educação (CBIE 2016)

Del Blanco, A. et al. (2010) "Easing Assessment of Game-based Learning with and LAMS", In ACM International Workshop on Multimedia Technologies for Distance Learning (MTDL 2010), In Conjunction with the ACM International Conference on Multimedia (In press). Firence, Italy: ACM Press.

Gee, J. P. (2007) “Good Video Games And Good Learning”. Tashia Morgridge Professor of Reading. University of Wisconsin-Madison, Vol. 1, No. 1, BOOK 1.

Rupp, A.A., Gushta, M., Mislevy, R.J., \& Shaffer, D.W. (2010) "Evidence-centered design of epistemic games: Measurement principles for complex learning environments", In: Journal of Technology, Learning, and Assessment.

Shaffer, D.W. (2006) "Epistemic frames for epistemic games", In: Computers and Education, 46, 223-234. 